\title{
Resistance-guided discovery of new antibiotics
}

Natural product screening, particularly of compounds derived from the Gram-positive bacteria actinomycetes, has been one of the most fruitful sources of antimicrobials. However, the 'low-hanging fruit' appear to have been picked. Now, reporting in Nature Biotechnology, the group of Gerry Wright demonstrates how the selfprotection mechanism of antibiotic producers can be exploited for the systematic discovery of new antibiotic scaffolds and their associated biosynthetic enzymes.

The scaffolds of about $80 \%$ of antibiotics approved for clinical use over the past decade were derived from microbial natural products. However, traditional screens of natural product extracts for compounds with antibiotic activity are faced with low hit rates and the problem of dereplication - the rediscovery of known compounds. For these reasons, the drug discovery sector turned increasingly to highthroughput screens of synthetic compounds, but such efforts were largely unsuccessful.

With the emergence of synthetic biology, which relies on access to libraries of antibiotic scaffolds and the associated biosynthetic machinery encoded in the genome of the producing strain, interest in natural-product-based antibiotic discovery has been revitalized.
However, identifying microbes with the biosynthetic capacity to produce desired compound classes involves the screening of metagenomic libraries - a technically demanding and time-intensive enterprise. For compound classes such as glycopeptide antibacterials (GPAs), for which synthetic biology approaches could be particularly useful in expanding diversity through chemical modifications, it has been estimated that $\sim 150,000$ actinomycete strains need to be screened to identify a single GPA producer.

Based on the fact that antibiotic producers need to evolve a selfprotection mechanism to avoid suicide, the authors reasoned that antibiotic selection could be a filter to identify antibiotic-producing bacteria. Proof of concept was generated in a screen for actinomycete strains that are resistant to the GPA vancomycin. Out of 1,000 randomly selected strains from an actinomycete library, 39 were found to be vancomycinresistant, and one of these carried biosynthetic genes for the production of GPAs. When the resistance filter was applied to a mixture of bacteria isolated directly from soil, the frequency of GPA producers among the resistant strains increased to about 1 in 10 .

Further PCR-based analysis of the biosynthetic genes provided a 'fingerprint' that allowed the classification of the type of GPA produced by the individual strains. This strategy led to the discovery of a new GPA, which was named peskiskomycin, with a rare scaffold and novel chemical modifications. It had modest antibacterial activity, but some effect against vancomycin-resistant enterococci type B.

The authors also successfully applied a similar approach (using different resistance filters) to facilitate the discovery of new members of other classes of antibiotics. By establishing phylogenetic trees of the respective biosynthetic clusters, they were able to predict relatedness to previously known antibiotics, thus avoiding dereplication and allowing the prioritization of the discovery of new scaffolds. Moreover, the strategy of preselection coupled with phylogenetic analysis allowed the discovery of latent producers - bacterial strains that produce only very low amounts of antibiotics.

Together, these results demonstrate a new strategy that should make the elusive 'high-hanging fruit' of novel natural-product-based antibiotics more accessible.

Alexandra Flemming

ORIGINAL RESEARCH PAPER Thaker, M. N. et al Identifying producers of antibacterial compounds by screening for antibiotic resistance. Nature Biotech. 31, 922-927 (2013) 